\title{
Who Gets a Post-Mortem? - 9 years experience in a tertiary Neonatal Intensive Care Unit
}

\author{
Bindal Desai and Vishna Rasiah
}

\section{Introduction}

Post mortem is the gold standard for diagnosis of death in neonates, infants and children. ${ }^{1}$ Post mortem not only offers a detailed examination into the cause of death of a neonate, it is also vital in providing statistics, public health monitoring as well as education, research and improving patient care. Despite this knowledge, the uptake of post mortems in neonates remains rather variable. ${ }^{1,2}$

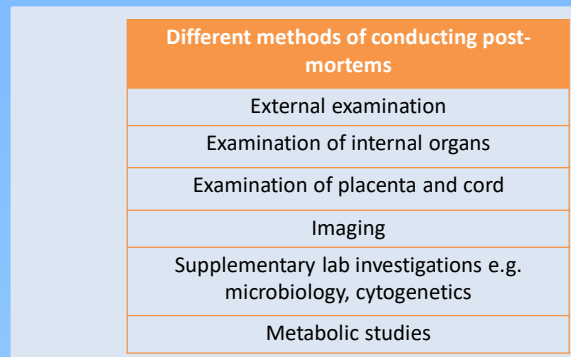

Table 1. Different methods of performing post mortems ${ }^{2}$

\section{Aim \\ The aim was to review all the post-mortems performed in the last 9 years for the deaths in a tertiary Neonatal intensive care unit, Birmingham Women's Hospital}

\section{Methods}

All babies who had a post-mortem between 01/04/2009 and $31 / 03 / 2018$ were identified. Their BadgerNet patient record were interrogated and analysed using an excel spreadsheet. All termination of pregnancies were excluded

\section{Results}

Total number of deaths of neonates at Birmingham Women's Hospital between $1^{\text {st }}$ April 2009 and $31^{\text {st }}$ March 2018 was 358 , of which 62 neonates had a post mortem carried out.

- Median gestational age was 28 weeks

Ranging between 22 and 41 weeks gestation

- Median birth weight 998g

Ranging between $380-3800 \mathrm{~g}$

- 19 of the babies who had a post mortem were known or suspected to have a congenital abnormality antenatally

9 of these were congenital diaphragmatic hernias

- 15 of the 62 babies who had a post mortem were born outside of Birmingham Women's Hospital

- 4 of the 62 babies had a limited post mortem

\section{PM rates in different gestational ages}

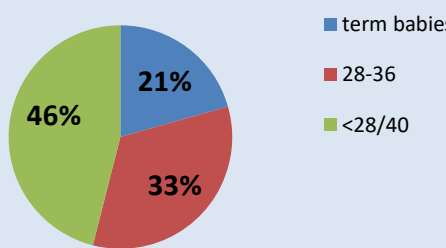

Figure 1. Proportion of post mortem rates in different gestational ages between Apr 2009 and Mar 2018

Proportion of term vs preterm babies having post mortems each financial year

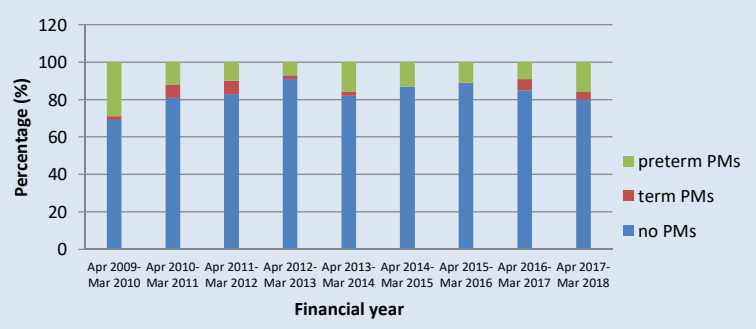

Figure 2. Proportion of post mortem rates in term vs preterm infants each financial year between Apr 2009 and Mar 2018

\section{Conclusions}

- $17.6 \%$ of babies that died at BWH had a post mortem over the 9 year period

- PM remains the gold standard examination in determining the cause of death.

- Despite this the uptake over the last 9 years remains rather low.

- Need to understand the reason behind this low uptake and support parents in pursuing a PM when their baby dies in NICU.

- Allow us to have a better understanding of the cause of death in their babies, help us learn and improve our practice.

\section{Discussion}

Why post mortem rates are low?

- Healthcare professionals relying on clinical findings and imaging 2,4

- Centralised post mortem services

- Parents poorly counselled

- Cultural background

How to improve post mortem rates?

- Presence of a pathologist at time of post mortem discussion

- Nursing specialist to have the discussion with parents and re-explore the decision

- Questionnaires to parents about why they consented or declined a post mortem ${ }^{5,6}$ 